\title{
EAl Endorsed Transactions

\section{Reliability-Security Analysis for Harvest-to-Jam based Multi-hop Cluster MIMO Networks Using Cooperative Jamming Methods Under Impact of Hardware Impairments}

\author{
Ngo The Anh ${ }^{1}$, Nguyen Canh Minh ${ }^{1}$, Tran Trung Duy ${ }^{2, *}$, Tan Hanh ${ }^{2}$, Hoang Dang Hai ${ }^{2}$ \\ ${ }^{1}$ University of Transport and Communications, Vietnam \\ ${ }^{2}$ Posts and Telecommunications Institute of Technology, Vietnam
}

\begin{abstract}
In this paper, we consider harvest-to-jam based secure multi-hop cluster multi-input multi-output networks, where a multi-antenna source sends its data to a multi-antenna destination via multi-antenna intermediate cluster heads. The data transmission at each hop is realized by using transmit antenna selection and selection combining techniques, and is overheard by a multi-antenna eavesdropper using selection combining. In addition, joint antenna and jammer selection methods are performed at each hop to reduce quality of the eavesdropping channels. The cluster members can harvest wireless energy from the previous cluster head, and use the harvested energy for emitting jamming noises on the eavesdropper. We propose three cooperative jamming algorithms, named best antenna and best jammer selection (BA-BJ), random antenna and all jammer selection (RA-AJ) and all antenna and all jammer selection (AA-AJ). Then, end-to-end outage probability and intercept probability of the proposed algorithms are evaluated via both simulation and analysis, under impact of hardware impairments, over Rayleigh fading channel.
\end{abstract}

Received on 28 August 2021; accepted on 13 September 2021; published on 17 September 2021

Keywords: Multi-hop cluster relaying networks, physical-layer security, harvest-to-jam technique, outage probability, intercept probability, TAS/SC.

Copyright (C) 2021 Ngo The Anh et al., licensed to EAI. This is an open access article distributed under the terms of the Creative Commons Attribution license, which permits unlimited use, distribution and reproduction in any medium so long as the original work is properly cited.

doi:10.4108/eai.17-9-2021.170963

\section{Introduction}

Wireless sensor networks (WSNs) [1-5] have been shown the widely applications in nearly all aspects of life nowadays. Tracking and monitoring are two main targets of WSNs, which cover almost applications including environment, agriculture, military, healthcare, and industry. The main components of WSNs are small sensor nodes (SNs) that are installed in the certain locations to sense data and information to send to the base station (sink) via radio links. There are three main units in a basic structure of SNs including transceiver unit, processing unit, and power unit [6]. The transceiver unit consists of the radio modem and the antenna to transmit/receive signals. The processing

*Corresponding author. Email: trantrungduy@ptithcm.edu.vn. unit consists of the microprocessor, the analogue to digital converter, the digital signal processing modem, the memory, and the sensors. The power unit is normally a replaceable battery. The simple structure and the small size of SNs bring the significant benefits for WSNs, such as quick deployment, low installation cost, high mobility, and smart infrastructure. However, the tiny size of SNs strongly affects the network lifetime of WSNs [6, 7]. The survey [7] has been proposed the number of techniques, which are efficiency resource allocation, opportunistic transmission, optimal routing, using mobile sensors (as relays) or mobile sinks to balance traffic in WSNs, optimizing the coverage and connectivity of SNs, applying energy harvesting (EH) strategy, and beam forming, to maximize the network lifetime of WSNs. Briefly, these solutions could be divided into three main groups: $\mathrm{EH}$ for maintaining the 
lifetime of SNs and prolonging the lifetime for WSNs; optimizing routing protocols to efficiently utilize the energy consumption of SNs, and multi-hop relaying communications to improve the performance of WSNs.

Due to the tiny size and the short communication range of SNs, the number of SNs in WSNs is normally large. Moreover, SNs are randomly deployed with the limited battery power to transport the sensed data from its source to the destination. This needs the efficient routing strategy for operations of WSNs. The chosen routing strategy applied to WSNs should ensure minimum energy consumption as battery replacement in sensors are often very difficult. Due to limited power, low bandwidth, no conventional addressing scheme, computational overheads and self-organization of SNs, designing an effective routing protocol is really a hard challenge. A lot of energy-efficient routing protocols have been proposed and developed for WSNs, depending on their application and network architecture [6-10]. In the survey of [8], there are four main schemes can be used to classify routing protocols in WSNs including Network Structure, Communication Model, Topology and Reliable Routing strategy. In the network structure scheme, the hierarchical routing protocol called LEACH (Low-Energy Adaptive Clustering Hierarchy) has been chosen to widely apply to WSNs. In LEACH, SNs can be divided into several groups of nodes called clusters. Then, each cluster is led by a cluster head $(\mathrm{CH})$, which is selected from SNs inside the cluster. $\mathrm{CH}$ collects the data from all of other nodes in the group to send to the destination sink. If $\mathrm{CH}$ is close enough to the $\mathrm{BS}$, then it can transmit the collected data to the BS directly via single hop; otherwise, the far $\mathrm{CH}$ has to send the data through the several intermediate nodes to the BS, following the multi-hop (MH) transmission model [10]. There are many successful LEACH protocols as shown in [10] including single-hop LEACH and MH LEACH. It can be said that LEACH is the most suitable routing protocol for WSNs, in term of power consumption utilization.

The MH relaying refers to the method of communications between source node (S) and destination node (D) via several relay nodes (R) [11-15]. Generally, $\mathrm{MH}$ significantly improves the power consumption, endto-end (e2e) throughput, and outage probability (OP) of the systems. In power consumption point of view, $\mathrm{MH}$ can help to reduce the consumed energy of all the nodes, due to the shorter range communication, which also improves the e2e throughput. However, the number of hops which strongly affects the e2e delay and throughput should be optimally designed. The authors in [16] studied the optimal number of hops to obtain better e2e throughput, in the presence of eavesdroppers. Although also studied MH wireless networks over Rayleigh fading channels to attain the energy efficiency,
Reference [17] optimized the power consumption with and without delay constraint in full-duplex (FD) systems. Different with [16, 17], Reference [18] considered the MH FD transmission systems over Nakagami- $m$ fading channels. The authors in [18] pointed out the considerable improvements in OP and power consumption. References $[19,20]$ proposed cooperative multi-hop transmission protocols which can reduce the number of hops by employing cooperative communication [21] under impact of hardware impairments (HIs). Because the protocols in $[19,20]$ required a high synchronization between all the nodes on the source-destination route and a high storage capacity at the relay nodes, the implementation is a very difficult work. Path-selection approaches were proposed in [22-24] to enhance the e2e OP of both MH multi-path decode-and-forward (DF) and amplify-and-forward (AF) relaying networks. However, complexity, high latency and outdated channel state information (CSI) problems are main disadvantages of these path-selection methods. To obtain the reliable transmission at each hop, relay selection methods in the cluster MH networks (or LEACH) were studied in $[25,26]$.

EH refers to the method, which can extract the energy from the natural sources such as solar, wind, vibration, and radio frequency (RF), then converts to the electrical energy for SNs. Due to the consistently availability of the RF signals, RF-EH is more flexible and sustainable than solar, wind, or vibration EH and then RF-EH becomes the most potential solution in terms of power supplying for SNs [27-30]. The RF signal not only carries the information but also supplies electrical power to the wireless nodes. Therefore, the base station in WSNs can transport both information and energy to SNs simultaneously. The authors in [28] studied the performance of WSNs in terms of OP, ergodic capacity, energy efficiency, and throughput of the links from the SNs to the sinks over Nakagami- $m$ fading channels. During the data transmission on these up-links, SNs have been powered by the RF-EH budget. The RF-EH strategy presented in [28] is either Time Splitting (TS) or Power Splitting (PS). Reference [28] also proposed an optimal splitting strategy for both TS and PS schemes to maximize the e2e throughput. Although achieved the significant results, the secrecy performance and the MH transmission have not been considered in [27-30]. These concerns have been studied in [31-34] to obtain security at the physical layer.

Due to the broadcast nature of wireless medium as well as the communication over multi-hop, the source data can be overheard by the eavesdroppers. Therefore, the security and privacy issues are the considerable challenges in WSNs. Conventionally, the original information will be ciphered by crypto codes before sending to the end users. This scheme coding is generally performed in the high-layer 
in the communication systems. However, due to computational complexity in the coded and de-coded algorithms, so it normally needs a large capacity of memories and storage, which may not be suitable for SNs. Recently, physical layer security (PLS) has been widely developed to satisfy the secrecy requirements, and also to reduce the computation in wireless communication systems [35-37]. Secrecy capacity that is an absolute difference between Shannon capacity of the data and eavesdropping channels is often used to measure the secrecy performance [35-41]. Different with [35-41], References [42, 43] evaluated intercept probability (IP) at the eavesdroppers in the PLS networks. In addition, the security-reliability trade-off (SRT) also investigated in $[42,43]$.

Cooperative jamming (CJ) $[44,45]$ is a key technique in PLS, where trusted nodes, often called jammers, are employed to emit artificial noises (ANs) on the eavesdroppers. Moreover, the jammers cooperate with the legitimate nodes so that the generated ANs can be removed at the legitimate receivers. References $[44,45]$ proposed joint relay and jammer selection methods to enhance the secrecy performance for the dual-hop relaying networks. In [45], the jammers have to harvest energy from the RF signals of the source for emitting ANs. In [46, 47], the authors evaluated secrecy outage performance for cooperative Non-Orthogonal Multiple Access (NOMA) using CJ. Reference [48] considered a randomize-and-forward strategy to confuse the eavesdropper, where the source and relay nodes use random code-books so that the eavesdropper cannot combine the received signals with maximal ratio combining (MRC). In addition, jammer selection and user-pair selection were proposed in [48] to enhance the SRT performance. Similar to [48], SRT with $\mathrm{CJ}$ and user-pair selection in two-way relaying networks was investigated in [49]. In addition, the authors of [49] considered a practical case of imperfect CSI estimation.

This paper concerns with multi-hop MIMO relaying in LEACH. Different with the conventional LEACH scenarios $[50,51], \mathrm{CHs}$ in our scheme are sink nodes which are equipped with multiple antennas, and they can use transmit antenna selection (TAS)/selection combining (SC) to hop-by-hop relay the source data to the destination. Cluster members (i.e., SNs) that are single-antenna nodes are employed to play the role of the cooperative jammers. Indeed, the jammers have to harvest the wireless energy from the previous $\mathrm{CH}$, and use the harvested energy to emit ANs. We propose three joint antenna selection and jammer selection algorithms to protect the data transmission at each hop. The most related works to this paper are [50-53], which also studied the e2e secrecy performance of the multi-hop cluster networks. However, different with our work, the schemes proposed in $[50,51]$ only considered the single-antenna $\mathrm{CHs}$ and random jammer selection. In other words, our proposed scheme is a generalized case of $[50,51]$. Unlike $[52,53]$, this paper evaluates the e2e IP and OP performance, while the authors in $[52,53]$ studied secrecy outage probability. Moreover, Reference [52] did not use the CJ technique, while the jammers in [53] harvested the RF energy from a power beacon deployed in the network.

In the following, we summarize main contribution obtained in this paper:

- We propose three joint antenna and jammer selection algorithms to enhance the e2e IP performance. In the first algorithm, the best antenna and the best jammer selection (BA-BJ) is proposed, relying on the channel gains between the $\mathrm{CHs}$ and jammer nodes. In the second one, named RA-AJ, a random antenna of $\mathrm{CH}$ is selected to supply the RF energy for the jammers, and all the jammers are selected for the CJ operation. In the last one, named AA-AJ, CHs use all the transmit antennas to support the energy for all the jammers which are employed to emit ANs on the eavesdropper.

- We derive the exact closed-form expressions of the e2e OP and IP for the BA-BJ, RA-AJ and AA-AJ algorithms, under the impact of HIs, over Rayleigh fading channel. The derived OP and IP expressions are then validated by Monte-Carlo simulations.

- We also compare the IP performance of three proposed algorithms, and investigate the SRT performance as well. In addition, the complexity of the proposed algorithms and the impact of the system parameters are also studied.

The rest of this paper is organized as follows. The system model is presented in Section 2. The OP and IP performance of the proposed algorithms is evaluated in Section 3. Section 4 points out the simulation results. Finally, Section 5 concludes the paper.

Notations: Expectation and probability operators are denoted by $\mathcal{E}\{$.$\} and \operatorname{Pr}($.$) , respectively. Cumulative$ Distribution Function (CDF) and Probability Density Function (PDF) of a random variable (RV) $X$ are denoted by $F_{X}($.$) and f_{X}($.$) , respectively. \Gamma(m, x)=$ $\int_{x}^{+\infty} t^{m-1} \exp (-t) d t$ is upper incomplete Gamma function [54], and $E_{1}(x)=\int_{x}^{+\infty} t^{-1} \exp (-t) d t$ is exponential integral function [54]. $C_{b}^{a}=b ! /[a !(b-a) !]$ denotes coefficient in Newton's binomial expansion.

\section{System Model}

In Fig. 1, the source node $\left(\mathrm{T}_{0}: \mathrm{CH}\right.$ of cluster 0$)$ attempts to send the data to the destination node $\left(\mathrm{T}_{M}: \mathrm{CH}\right.$ of 


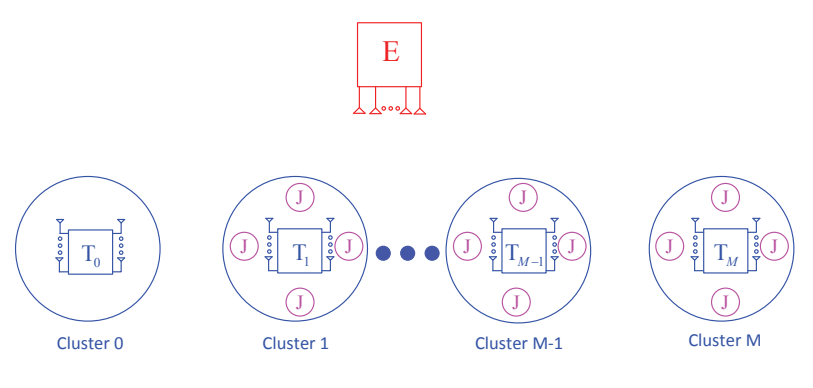

Figure 1. System model of the proposed harvest-to-jam based secure multi-hop cluster MIMO scheme.

cluster $M$ ). Because the direct link between $\mathrm{T}_{0}$ and $\mathrm{T}_{M}$ does not exist, the $\mathrm{T}_{0} \rightarrow \mathrm{T}_{M}$ transmission is realized via $\mathrm{CHs}$ of $(M-1)$ intermediate clusters, using the $\mathrm{MH}$ DF relaying model. In the $m$ - th cluster, we denote $\mathrm{T}_{m}$ as $\mathrm{CH}, K_{m}$ as number of cluster members, and $\mathrm{J}_{m, u}$ as a member, where $m \in[0, M], u \in\left[1, K_{m}\right]$. Also in Fig. 1 , the eavesdropper (E) overhears the data sent by the source and $\mathrm{CH}$ nodes. Without loss of generality, we assume that the number of antennas at all the $\mathrm{CHs}$ are $N_{\mathrm{T}}$, and that at $\mathrm{E}$ is $N_{\mathrm{E}}$. The cluster members $\mathrm{J}_{m, u}$ are $\mathrm{SNs}$, and they have only a single antenna. In addition, operating on a half-duplex mode, the data transmission between the source and the destination is split into orthogonal time slots, and the transmission between two adjacent nodes $\mathrm{T}_{m-1}$ and $\mathrm{T}_{m}$ is realized with TAS/SC. Also, the E node uses SC to decode the signals received at each hop. To protect the source data, the node $\mathrm{J}_{m, u}$ harvests the RF energy from $\mathrm{T}_{m-1}$ to emit ANs. Furthermore, the source and the intermediate $\mathrm{CHs}$ encode the data with random code-books, follows the randomize-and-forward strategy [50-53].

Let us denote $\mathcal{L}(\mathcal{L}$ is normalized by 01$)$ as the e2e delay of each $\mathrm{T}_{0} \rightarrow \mathrm{T}_{M}$ transmission, and hence duration allocated for each time slot is $\tau=\mathcal{L} / M=1 / M$. In addition, using the TS RF-EH approach [28], the $m$ - th time slot is also divided into two sub-time slots, i.e., the first one (duration of $\alpha \tau$ ) is used for $\mathrm{EH}$, and the second one (duration of $(1-\alpha) \tau)$ is used for the data transfer, where $0 \leq \alpha \leq 1$.

Assume that all the channels are Rayleigh fading, channel gain $\psi_{\mathrm{X}^{n_{t}} \mathrm{Y}^{n_{r}}}$ between the $n_{\mathrm{t}}$ - th antenna of the transmitter $\mathrm{X}$ and the $n_{\mathrm{r}}-$ th antenna of the receiver $\mathrm{Y}$ is an exponential RV, where $(\mathrm{X}, \mathrm{Y}) \in\left\{\mathrm{T}_{m}, \mathrm{~J}_{m, u}, \mathrm{E}\right\}, n_{t} \in$ $\left[1, N_{\mathrm{X}}\right], n_{r} \in\left[1, N_{\mathrm{Y}}\right]$. As a result, CDF and PDF of $\psi_{\mathrm{X}^{n_{t}} \mathrm{Y}^{n_{r}}}$ are given, respectively as

$$
\begin{aligned}
F_{\psi_{\mathrm{X}^{n} \mathrm{Y}^{n_{r}}}}(x) & =1-\exp \left(-\Omega_{\mathrm{XY}} x\right), \\
f_{\psi_{\mathrm{X}^{n} \mathrm{Y}^{n_{r}}}}(x) & =\Omega_{\mathrm{XY}} \exp \left(-\Omega_{\mathrm{XY}} x\right),
\end{aligned}
$$

where $\Omega_{\mathrm{XY}}=1 / \mathcal{E}\left\{\psi_{\mathrm{X}^{n} \mathrm{Y}^{n_{r}}}\right\}=d_{\mathrm{XY}}^{\beta}[21], d_{\mathrm{XY}}$ is the $\mathrm{X}-\mathrm{Y}$ distance, and $\beta$ is a path-loss exponent.
Remark 1: Since the nodes $\mathrm{T}_{m}$ and $\mathrm{J}_{m, u}$ are close together, we can assume that $\Omega_{\mathrm{T}_{m-1} \mathrm{~J}_{m, u}}=\Omega_{\mathrm{T}_{m-1} \mathrm{~T}_{m}}$, $\Omega_{\mathrm{J}_{m, u} \mathrm{E}}=\Omega_{\mathrm{T}_{m} \mathrm{E}}$, for all $m$ and $u$.

\subsection{Joint Antenna Selection and Jammer Selection Algorithms}

In BA-BJ, $\mathrm{T}_{m-1}$ selects one of its available antennas to support the energy for one of members of the $m$ - th cluster. Mathematically speaking, the BA-BJ algorithm can be written as

$$
\psi_{\mathrm{T}_{m-1}^{a} \mathrm{~J}_{m, b}}=\max _{r=1, \ldots, N_{\mathrm{T}}}\left(\max _{u=1, \ldots, K_{m}}\left(\psi_{\mathrm{T}_{m-1}^{r} \mathrm{~J}_{m, u}}\right)\right),
$$

where $a$ is index of the selected antenna at $\mathrm{T}_{m-1}$, and $\mathrm{J}_{m, b}$ is the chosen node of the $m$ - th cluster. Equation (2) implies that $T_{m-1}$ attempts to provide the highest harvested energy for the selected jammer. Next, the energy that $\mathrm{J}_{m, b}$ can harvest from $\mathrm{T}_{m-1}$ during the $\mathrm{EH}$ phase can be formulated as in $[27,28]$ :

$$
E_{\mathrm{J}_{m, b}}=\eta \alpha \tau P \psi_{\mathrm{T}_{m-1}^{a} \mathrm{~J}_{m, b}},
$$

where $\eta(0 \leq \eta \leq 1)$ is energy conversion efficiency, $P$ is transmit power of $\mathrm{T}_{m-1}$ for all $m$. Hence, the average transmit power of $\mathrm{J}_{m, b}$ during the data transfer phase is given by

$$
P_{\mathrm{J}_{m, b}}=\frac{E_{\mathrm{J}_{m, b}}}{(1-\alpha) \tau}=\kappa P \psi_{\mathrm{T}_{m-1}^{a} \mathrm{~J}_{m, b}},
$$

where $\kappa=\eta \alpha /(1-\alpha)$.

In RA-AJ, all the nodes $\mathrm{J}_{m, u}$ are selected for the CJ operation, and $\mathrm{T}_{m-1}$ randomly selects one antenna to send the energy to them. Similar to (3)-(4), the average transmit power of $\mathrm{J}_{m, u}$ in RA-AJ is expressed as

$$
P_{\mathrm{J}_{m, u}}=\kappa P \psi_{\mathrm{T}_{m-1}^{c} \mathrm{~J}_{m, u}},
$$

where $c$ is index of the selected antenna at $\mathrm{T}_{m-1}$.

In AA-AJ, $\mathrm{T}_{m-1}$ uses all the antennas to support the energy for all the $\mathrm{J}_{m, u}$ nodes. Therefore, the average transmit power of $\mathrm{J}_{m, u}$ in AA-AJ can be formulated as

$$
P_{\mathrm{J}_{m, u}}=\kappa P \sum_{r=1}^{N_{\mathrm{T}}} \psi_{\mathrm{T}_{m-1}^{r} \mathrm{~J}_{m, u}} .
$$

Remark 2: It is reminded that since $\mathrm{CHs}$ and their members are close together, they can securely exchange information about ANs. As a result, the $\mathrm{T}_{m}$ receiver can remove the interference components caused by $\mathrm{J}_{m, u}$ (while the E node cannot). Next, the implementation of RA-AJ and AA-AJ is more complex than that of BA$B J$ because it requires high synchronization between $\mathrm{T}_{m}$ and its all members to perform the interference cancellation. In addition, the implementation of AA-AJ is the most complex because it spends more energy and resources (e.g., all the antennas) for the $\mathrm{EH}$ phase. 


\subsection{TAS and SC Techniques}

At the $m$ - th time slot, the $\mathrm{T}_{m-1} \rightarrow \mathrm{T}_{m}$ transmission is performed by using TAS/SC [52], i.e.,

$$
\psi_{\mathrm{T}_{m-1}^{p} \mathrm{~T}_{m}^{q}}=\max _{u=1, \ldots, N_{\mathrm{T}}}\left(\max _{v=1, \ldots, N_{\mathrm{T}}}\left(\psi_{\mathrm{T}_{m-1}^{u} \mathrm{~T}_{m}^{v}}\right)\right),
$$

where $p$ and $q$ are indices of the transmit and receive antennas selected by $\mathrm{T}_{m-1}$ and $\mathrm{T}_{m}$, respectively. When the interference cancellation is perfect; the instantaneous channel capacity of the $\mathrm{T}_{m-1} \rightarrow \mathrm{T}_{m}$ link, under the presence of hardware imperfection, can be given as

$$
\begin{aligned}
C_{\mathrm{D}, m} & =(1-\alpha) \tau \log _{2}\left(1+\frac{P \psi_{\mathrm{T}_{m-1}^{p} \mathrm{~T}_{m}^{q}}}{\mu_{\mathrm{D}}^{2} P \psi_{\mathrm{T}_{m-1}^{p} \mathrm{~T}_{m}^{q}+\sigma_{0}^{2}}}\right) \\
& =(1-\alpha) \tau \log _{2}\left(1+\frac{\Delta \psi_{\mathrm{T}_{m-1}^{p}} \mathrm{~T}_{m}^{q}}{\mu_{\mathrm{D}}^{2} \Delta \psi_{\mathrm{T}_{m-1}^{p}}^{p} \mathrm{~T}_{m}^{q}+1}\right),
\end{aligned}
$$

where $\sigma_{0}^{2}$ is variance of Additive White Gaussian Noise (AWGN), $\Delta=P / \sigma_{0}^{2}$ is transmit SNR, $\mu_{\mathrm{D}}^{2}$ is total HI level of the $\mathrm{T}_{m-1} \rightarrow \mathrm{T}_{m}$ link $[19,20]$, for all $m$. Due to the DF relaying method, the e2e channel capacity can be obtained as

$$
C_{\mathrm{D}}^{\mathrm{e} 2 \mathrm{e}}=\min _{m=1,2, \ldots, M}\left(C_{\mathrm{D}, m}\right)
$$

For the eavesdropper node, the SC technique at the $m$ - th time slot can be written as

$$
\psi_{\mathrm{T}_{m-1}^{p} \mathrm{E}^{r}}=\max _{v=1, \ldots, N_{\mathrm{E}}}\left(\psi_{\mathrm{T}_{m-1}^{p} \mathrm{E}^{v}}\right),
$$

where $r$ denotes index of the receive antenna selected by E. Under the joint impact of ANs and HIs, the obtained signal-to-interference-plus-noise (SINR) by E, at the $m$ - th time slot, in the BA-BJ, RA-AJ and AA-AJ algorithms, can be formulated, respectively as

$$
\begin{aligned}
\varphi_{\mathrm{T}_{m-1}^{p} \mathrm{E}^{r}}^{\mathrm{BA}-\mathrm{BJ}} & =\frac{\Delta \psi_{\mathrm{T}_{m-1}^{p} \mathrm{E}^{r}}}{\mu_{\mathrm{E}}^{2} \Delta \psi_{\mathrm{T}_{m-1}^{p} \mathrm{E}^{r}}+\kappa \Delta \psi_{\mathrm{T}_{m-1}^{a} \mathrm{~J}_{m, b}} \psi_{\mathrm{J}_{m, b} \mathrm{E}^{r}+1}}, \\
\varphi_{\mathrm{T}_{m-1}^{p} \mathrm{E}^{r}}^{\mathrm{RA}-\mathrm{AJ}} & =\frac{\Delta \psi_{\mathrm{T}_{m-1}^{p} \mathrm{E}^{r}}^{K_{m}}}{\mu_{\mathrm{E}}^{2} \Delta \psi_{\mathrm{T}_{m-1}^{p} \mathrm{E}^{r}}+\kappa \Delta \sum_{u=1}^{\psi_{\mathrm{T}_{m-1}}^{c} \mathrm{~J}_{m, u}} \psi_{\mathrm{J}_{m, u}} \mathrm{E}^{r}+1}, \\
\varphi_{\mathrm{T}_{m-1}^{p} \mathrm{E}^{r}}^{\mathrm{AA}-\mathrm{AJ}} & =\frac{\Delta \psi_{\mathrm{T}_{m-1}^{p} \mathrm{E}^{r}}}{\mu_{\mathrm{E}}^{2} \Delta \psi_{\mathrm{T}_{m-1}^{p} \mathrm{E}^{r}}+\kappa \Delta \sum_{t=1}^{N_{\mathrm{T}}} \sum_{u=1}^{K_{m}} \psi_{\mathrm{T}_{m-1}^{t} \mathrm{~J}_{m, u}} \psi_{\mathrm{J}_{m, u}} \mathrm{E}^{r}+1},
\end{aligned}
$$

where $\mu_{\mathrm{E}}^{2}$ is total HI level of the $\mathrm{T}_{m-1} \rightarrow \mathrm{E}$ link, for all $m$. From (11)-(13), the instantaneous channel capacity obtained at $\mathrm{E}$ in the $m$ - th time slot can be written as

$$
C_{\mathrm{E}, m}^{\mathrm{Z}}=(1-\alpha) \tau \log _{2}\left(1+\varphi_{\mathrm{T}_{m-1}^{p} \mathrm{E}^{r}}^{\mathrm{Z}}\right)
$$

where $Z \in\{B A-B J, R A-A J, A A-A J\}$. Then, the e2e eavesdropping rate can be given as in [50]:

$$
C_{\mathrm{E}}^{\mathrm{e} 2 \mathrm{e}, \mathrm{Z}}=\max _{m=1,2, \ldots, M}\left(C_{\mathrm{E}, m}^{\mathrm{Z}}\right)
$$

Remark 3: It is noted from (11)-(13) that the eavesdropper cannot select the receive antenna providing the highest SINR because it cannot know the transmit power of the jammer nodes.

\subsection{Definition of $\mathrm{OP}$ and IP}

The e2e OP can be defined as

$$
\begin{aligned}
\mathrm{OP}_{\mathrm{e} 2 \mathrm{e}} & =\operatorname{Pr}\left(C_{\mathrm{D}}^{\mathrm{e} 2 \mathrm{e}}<C_{\mathrm{th}}\right) \\
& =1-\prod_{m=1}^{M}\left(1-\operatorname{Pr}\left(C_{\mathrm{D}, m}<C_{\mathrm{th}}\right)\right) \\
& =1-\prod_{m=1}^{M}\left(1-\mathrm{OP}_{m}\right),
\end{aligned}
$$

where $C_{\text {th }}$ is a pre-determined target rate, and $\mathrm{OP}_{m}=\operatorname{Pr}\left(C_{\mathrm{D}, m}<C_{\mathrm{th}}\right)$ is OP at the $m$-th time slot. Next, the e2e IP of the $Z$ algorithm, $Z \in$ $\{\mathrm{BA}-\mathrm{BJ}, \mathrm{RA}-\mathrm{AJ}, \mathrm{AA}-\mathrm{AJ}\}$, is defined as follows:

$$
\begin{aligned}
\mathrm{IP}_{\mathrm{Z}}^{\mathrm{e} 2 \mathrm{e}} & =\operatorname{Pr}\left(C_{\mathrm{E}}^{\mathrm{e} 2 \mathrm{e}, \mathrm{Z}} \geq C_{\mathrm{th}}\right) \\
& =1-\prod_{m=1}^{M} \operatorname{Pr}\left(C_{\mathrm{E}, m}^{\mathrm{Z}}<C_{\mathrm{th}}\right) \\
& =1-\prod_{m=1}^{M} \overline{\mathrm{IP}_{m}^{Z}},
\end{aligned}
$$

where $\overline{\mathrm{IP}_{m}^{Z}}=\operatorname{Pr}\left(C_{\mathrm{E}, m}^{\mathrm{Z}}<C_{\mathrm{th}}\right)$ is the probability that the eavesdropper $\mathrm{E}$ cannot correctly decode the data at the $m$ - th time slot.

\section{Performance Analysis}

In this section, exact and asymptotic closed-form expressions of the e2e OP and IP of the BA-BJ, RA-AJ and AA-AJ algorithms over Rayleigh fading channel are derived.

\subsection{E2e OP}

Using (8), we can write OP at the time slot as

$$
\mathrm{OP}_{m}=\operatorname{Pr}\left(\left(1-\mu_{\mathrm{D}}^{2} \rho_{\mathrm{th}}\right) \Delta \psi_{\mathrm{T}_{m-1}^{p}} \mathrm{~T}_{m}^{q}<\rho_{\mathrm{th}}\right),
$$


where

$$
\rho_{\mathrm{th}}=2^{C_{\mathrm{th}} /(1-\alpha) \tau}-1 .
$$

We can observe from (18) that $\mathrm{OP}_{m}=1$ when $1-$ $\mu_{\mathrm{D}}^{2} \rho_{\text {th }} \leq 0$. Otherwise, from (1) and (7), we have

$$
\begin{aligned}
& \mathrm{OP}_{m}=\operatorname{Pr}\left(\psi_{\mathrm{T}_{m-1}^{p} \mathrm{~T}_{m}^{q}}<\frac{\rho_{\mathrm{th}}}{\left(1-\mu_{\mathrm{D}}^{2} \rho_{\mathrm{th}}\right) \Delta}\right)
\end{aligned}
$$

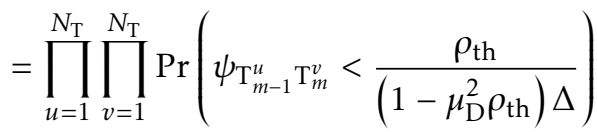

$$
\begin{aligned}
& =\left[1-\exp \left(-\frac{\Omega_{\mathrm{T}_{m-1} \mathrm{~T}_{m}} \rho_{\mathrm{th}}}{\left(1-\mu_{\mathrm{D}}^{2} \rho_{\mathrm{th}}\right) \Delta}\right)\right]^{N_{\mathrm{T}}^{2}} \text {. }
\end{aligned}
$$

Furthermore, at high transmit $\operatorname{SNR}(\Delta)$, equation (20) can be approximated as

$$
\mathrm{OP}_{m} \stackrel{\Delta \rightarrow+\infty}{\approx}\left[\frac{\Omega_{\mathrm{T}_{m-1}} \mathrm{~T}_{m} \rho_{\mathrm{th}}}{\left(1-\mu_{\mathrm{D}}^{2} \rho_{\mathrm{th}}\right) \Delta}\right]^{N_{\mathrm{T}}^{2}}
$$

Remark 4: Substituting (20) into (16), we obtain the exact closed-form formula of the e2e OP when $1-\mu_{\mathrm{D}}^{2} \rho_{\mathrm{th}}>0$. It is straightforward that if $1-\mu_{\mathrm{D}}^{2} \rho_{\mathrm{th}} \leq$ 0 , then $\mathrm{OP}_{\mathrm{e} 2 \mathrm{e}}=1$. Moreover, all the proposed BA-BJ, RA-AJ, AA-AJ algorithms have the same e2e OP value. Finally, at high transmit SNR, using (20), we obtain the asymptotic formula of $\mathrm{OP}_{\mathrm{e} 2 \mathrm{e}}$ as follows:

$$
\begin{aligned}
\mathrm{OP}_{\mathrm{e} 2 \mathrm{e}} \stackrel{\Delta \rightarrow+\infty}{\underset{\approx}{\approx}} \sum_{m=1}^{M} \mathrm{OP}_{m} \\
\stackrel{\Delta \rightarrow+\infty}{\approx} \sum_{m=1}^{M}\left[\frac{\Omega_{\mathrm{T}_{m-1} \mathrm{~T}_{m}} \rho_{\mathrm{th}}}{\left(1-\mu_{\mathrm{D}}^{2} \rho_{\mathrm{th}}\right) \Delta}\right]^{N_{\mathrm{T}}^{2}} .
\end{aligned}
$$

Using (22), Diversity Gain (DG) is calculated as

$$
\mathrm{DG}=-\lim _{\Delta \rightarrow+\infty} \frac{\log \left(\mathrm{OP}_{\mathrm{e} 2 \mathrm{e}}\right)}{\log (\Delta)}=N_{\mathrm{T}}^{2} .
$$

\subsection{E2e IP}

Firstly, we rewrite $\overline{\mathrm{IP}_{m}^{Z}}$ under the following form:

$$
\overline{\mathrm{IP}_{m}^{Z}}=\operatorname{Pr}\left(\varphi_{\mathrm{T}_{m-1}^{p} \mathrm{E}^{r}}^{\mathrm{Z}}<\rho_{\mathrm{th}}\right) .
$$

Considering BA-BJ; substituting (11) into (24), which yields

$$
\begin{aligned}
& \overline{\mathrm{IP}}_{m}^{\mathrm{BA}-\mathrm{BJ}}=\operatorname{Pr}\left(\frac{\Delta \psi_{\mathrm{T}_{m-1}^{p} \mathrm{E}^{r}}}{\mu_{\mathrm{E}}^{2} \Delta \psi_{\mathrm{T}_{m-1}^{p}} \mathrm{E}^{r}+\kappa \Delta \psi_{\mathrm{T}_{m-1}^{a} \mathrm{~J}_{m, b}} \psi_{\mathrm{J}_{m, b} \mathrm{E}^{r}+1}}<\rho_{\mathrm{th}}\right) \\
& =\operatorname{Pr}\left(\left(1-\mu_{\mathrm{E}}^{2} \rho_{\mathrm{th}}\right) \Delta \psi_{\mathrm{T}_{m-1}^{p} \mathrm{E}^{r}}<\kappa \rho_{\mathrm{th}} \Delta \psi_{\mathrm{T}_{m-1}^{a} \mathrm{~J}_{m, b}} \psi_{\mathrm{J}_{m, b}} \mathrm{E}^{r}+\rho_{\mathrm{th}}\right) .
\end{aligned}
$$

It is straightforward that if $1-\mu_{\mathrm{E}}^{2} \rho_{\text {th }} \leq 0$, then $\overline{\mathrm{IP}}_{m}^{\mathrm{BA}-\mathrm{BJ}}=1$. In the following, we only consider the case where $1-\mu_{\mathrm{E}}^{2} \rho_{\text {th }}>0$.

Next, we can rewrite (25) as

$$
\begin{gathered}
\overline{\mathrm{IP}}_{m}^{\mathrm{BA}-\mathrm{BJ}}=\operatorname{Pr}\left(\psi_{\mathrm{T}_{m-1}^{p} \mathrm{E}^{r}}<\omega_{1} \psi_{\mathrm{T}_{m-1}^{a} \mathrm{~J}_{m, b}} \psi_{\mathrm{J}_{m, b} \mathrm{E}^{r}}+\omega_{2}\right) \\
=\int_{0}^{+\infty} \int_{0}^{+\infty} F_{\psi_{\mathrm{T}_{m-1}^{p}}^{p} \mathrm{E}^{r}}\left(\omega_{1} x y+\omega_{2}\right) f_{\psi_{\mathrm{J}_{m, b}} \mathrm{E}^{r}}(x) \\
\times f_{\psi_{\mathrm{T}_{m-1}^{a} \mathrm{~J}_{m, b}}}(y) d x d y,
\end{gathered}
$$

where

$$
\omega_{1}=\frac{\kappa \rho_{\mathrm{th}}}{1-\mu_{\mathrm{E}}^{2} \rho_{\mathrm{th}}}, \omega_{2}=\frac{\rho_{\mathrm{th}}}{\left(1-\mu_{\mathrm{E}}^{2} \rho_{\mathrm{th}}\right) \Delta} .
$$

From (1) and (10), we can obtain CDF of $\psi_{\mathrm{T}_{m-1}^{p} \mathrm{E}^{r}}$ as

$$
\begin{aligned}
& F_{\psi_{\mathrm{T}_{m-1}^{p}}^{p} \mathrm{E}^{r}}(x)=\prod_{v=1}^{N_{\mathrm{E}}} F_{\mathrm{T}_{m-1}^{p} \mathrm{E}^{v}}(x) \\
& =\left(1-\exp \left(-\Omega_{\mathrm{T}_{m-1} \mathrm{E}} x\right)\right)^{N_{\mathrm{E}}} \\
& =1+\sum_{p=1}^{N_{\mathrm{E}}}(-1)^{p} C_{N_{\mathrm{E}}}^{p} \exp \left(-p \Omega_{\mathrm{T}_{m-1} \mathrm{E}} x\right) .
\end{aligned}
$$

Substituting (28) and PDF $f_{\psi_{\mathrm{J}_{m, b} \mathrm{E}^{r}}}(x)=$ $\Omega_{\mathrm{T}_{m} \mathrm{E}} \exp \left(-\Omega_{\mathrm{T}_{m} \mathrm{E}} x\right)$ (see (1) and Remark 1) into (26), after some algebraic manipulation, we have

$$
\begin{aligned}
\overline{\mathrm{IP}}_{m}^{\mathrm{BA}-\mathrm{BJ}}=1+ & \sum_{p=1}^{N_{\mathrm{E}}}(-1)^{p} C_{N_{\mathrm{E}}}^{p} \exp \left(-p \Omega_{\mathrm{T}_{m-1} \mathrm{E}} \omega_{2}\right) \\
& \times \int_{0}^{+\infty} \frac{\theta_{1, p}}{\theta_{1, p}+y} f_{\psi_{\mathrm{T}_{m-1}^{a} \mathrm{~J}_{m, b}}}(y) d y,
\end{aligned}
$$

where $\theta_{1, p}=\Omega_{\mathrm{T}_{m} \mathrm{E}} /\left(p \Omega_{\mathrm{T}_{m-1} \mathrm{E}} \omega_{1}\right)$.

Moreover, from (1)-(2) and Remark 1, CDF of $\psi_{\mathrm{T}_{m-1}^{a} \mathrm{~J}_{m, b}}$ can be expressed as

$$
\begin{aligned}
F_{\psi_{\mathrm{T}_{m-1}^{a} \mathrm{~J}_{m, b}}}(y) & =\prod_{r=1}^{N_{\mathrm{T}}} \prod_{u=1}^{K_{m}} F_{\psi_{\mathrm{T}_{m-1}^{r} \mathrm{~J}_{m, u}}}(y) \\
& =\left[1-\exp \left(-\Omega_{\mathrm{T}_{m-1} \mathrm{~T}_{m}} y\right)\right]^{N_{\mathrm{T}} K_{m}} .
\end{aligned}
$$

Then, the corresponding PDF is

$$
\begin{aligned}
f_{\psi_{\mathrm{T}_{m-1}} \mathrm{I}_{m, b}}(y) & =N_{\mathrm{T}} K_{m} \Omega_{\mathrm{T}_{m-1} \mathrm{~T}_{m}} \exp \left(-\Omega_{\mathrm{T}_{m-1} \mathrm{~T}_{m}} y\right) \\
& \times\left[1-\exp \left(-\Omega_{\mathrm{T}_{m-1} \mathrm{~T}_{m}} y\right)\right]^{N_{\mathrm{T}} K_{m}-1} \\
& =\sum_{u=0}^{N_{\mathrm{T}} K_{m}-1}(-1)^{u} C_{N_{\mathrm{T}} K_{m}-1}^{u} N_{\mathrm{T}} K_{m} \Omega_{\mathrm{T}_{m-1} \mathrm{~T}_{m}} \\
& \times \exp \left(-(u+1) \Omega_{\mathrm{T}_{m-1} \mathrm{~T}_{m}} y\right) .
\end{aligned}
$$


Substituting (31) into (29), after some calculation, we obtain

$$
\begin{aligned}
& \overline{\mathrm{IP}}_{m}^{\mathrm{BA}-\mathrm{BJ}}=1 \\
& +\sum_{p=1}^{N_{\mathrm{E}}} \sum_{u=0}^{N_{\mathrm{T}} K_{m}-1}(-1)^{p+u} C_{N_{\mathrm{E}}}^{p} C_{N_{\mathrm{T}} K_{m}-1}^{u} N_{\mathrm{T}} K_{m} \\
& \times \exp \left((u+1) \Omega_{\mathrm{T}_{m-1} \mathrm{~T}_{m}} \theta_{1, p}-p \Omega_{\mathrm{T}_{m-1} \mathrm{E}} \omega_{2}\right) \\
& \times \Omega_{\mathrm{T}_{m-1} \mathrm{~T}_{m}} \theta_{1, p} E_{1}\left((u+1) \Omega_{\mathrm{T}_{m-1} \mathrm{~T}_{m}} \theta_{1, p}\right) .
\end{aligned}
$$

Combining (17) and (32), the e2e IP of BA-BJ can be exactly expressed as in (33) at the top of next page.

Considering high transmit SNR regimes, we have

$$
\begin{aligned}
& \frac{\Delta \psi_{\mathrm{T}_{m-1}^{p}} \mathrm{E}^{r}}{\mu_{\mathrm{E}}^{2} \Delta \psi_{\mathrm{T}_{m-1}^{p}} \mathrm{E}^{r}+\kappa \Delta \psi_{\mathrm{T}_{m-1}^{a}}^{a} \mathrm{~J}_{m, b} \psi_{\mathrm{J}_{m, b}} \mathrm{E}^{r}+1} \\
& \stackrel{\Delta \rightarrow+\infty}{\approx} \frac{\psi_{\mathrm{T}_{m-1}^{p}}^{p} \mathrm{E}^{r}}{\mu_{\mathrm{E}}^{2} \psi_{\mathrm{T}_{m-1}^{p}} \mathrm{E}^{r}+\kappa \psi_{\mathrm{T}_{m-1}^{a} \mathrm{~J}_{m, b}} \psi_{\mathrm{J}_{m, b} \mathrm{E}^{r}}} .
\end{aligned}
$$

From (34), with the same manner as deriving (33), $\mathrm{IP}_{\mathrm{e} 2 \mathrm{e}}^{\mathrm{BA}-\mathrm{BJ}}$ can be approximated as given in (35) at the top of next page.

For RA-AJ, with $1-\mu_{\mathrm{E}}^{2} \rho_{\text {th }}>0$ and (11), similar to (26) and (29), we can write $\overline{\mathrm{IP}}_{m}^{\mathrm{RA}-\mathrm{AJ}}$ as (36) (see the next page).

Substituting $\quad f_{\psi_{\mathrm{J}_{m, u}} \mathrm{E}^{r}}(x)=\Omega_{\mathrm{T}_{m} \mathrm{E}} \exp \left(-\Omega_{\mathrm{T}_{m} \mathrm{E}} x\right)$ and $f_{\psi_{\mathrm{T}_{m-1}^{c} \mathrm{~J}_{m, u}}}(y)=\Omega_{\mathrm{T}_{m-1} \mathrm{~T}_{m}} \exp \left(-\Omega_{\mathrm{T}_{m-1} \mathrm{~T}_{m}} y\right)$ into (36), after some manipulation, we obtain

$$
\begin{aligned}
& \overline{\mathrm{IP}}_{m}^{\mathrm{RA}-\mathrm{AJ}}=1+ \\
& \sum_{p=1}^{N_{\mathrm{E}}}(-1)^{p} C_{N_{\mathrm{E}}}^{p} \exp \left(K_{m} \Omega_{\mathrm{T}_{m-1} \mathrm{~T}_{m}} \theta_{1, p}-p \Omega_{\mathrm{T}_{m-1} \mathrm{E}} \omega_{2}\right) \\
& \times\left[\Omega_{\mathrm{T}_{m-1} \mathrm{~T}_{m}} \theta_{1, p} E_{1}\left((p+1) \Omega_{\mathrm{T}_{m-1} \mathrm{~T}_{m}} \theta_{1, p}\right)\right]^{K_{m}} .
\end{aligned}
$$

Combining (17) and (37), the e2e IP of RA-AJ can be given by (38) at the next page. Similarly, at high transmit SNR, we can approximate $\mathrm{IP}_{\mathrm{e} 2 \mathrm{e}}^{\mathrm{RA}-\mathrm{AJ}}$ as in (39) at the next page.

For AA-AJ, $\overline{\mathrm{IP}}_{m}^{\mathrm{AA}-\mathrm{AJ}}$ is also given by (40) at the next page, where $Y_{\text {sum }}=\sum_{t=1}^{N_{\mathrm{T}}} \psi_{\mathrm{T}_{m-1}^{t} \mathrm{~J}_{m, u}}$.

In addition, since $Y_{\text {sum }}$ is summation of independently and identically exponential RVs, its PDF can be obtained as (see [55])

$$
f_{Y_{\text {sum }}}(x)=\frac{\Omega_{\mathrm{T}_{m-1} \mathrm{~T}_{m}}^{N_{\mathrm{T}}}}{\left(N_{\mathrm{T}}-1\right) !} y^{N_{\mathrm{T}}-1} \exp \left(-\Omega_{\mathrm{T}_{m-1} \mathrm{~T}_{m}} y\right) .
$$

Next, substituting (41) into (40), and after interchanging variable $z=\theta_{1}+y$, we obtain (42) (see the next page).
Out next objective is to calculate the integral $\mathcal{I}$ as marked in (42). Using binomial expansion for $\left(z-\theta_{1, p}\right)^{N_{\mathrm{T}}-1}$, we have

$$
\begin{aligned}
\mathcal{I} & =\sum_{q=0}^{N_{\mathrm{T}}-1}(-1)^{q} C_{N_{\mathrm{T}}-1}^{q}\left(\theta_{1, p}\right)^{q} \\
& \times \int_{\theta_{1, p}}^{+\infty} z^{N_{\mathrm{T}}-2-q} \exp \left(-\Omega_{\mathrm{T}_{m-1} \mathrm{~T}_{m}} z\right) d z \\
& =(-1)^{N_{\mathrm{T}}-1}\left(\theta_{1, p}\right)^{N_{\mathrm{T}}-1} E_{1}\left(\Omega_{\mathrm{T}_{m-1} \mathrm{~T}_{m}} \theta_{1, p}\right) \\
& +\sum_{q=0}^{N_{\mathrm{T}}-2}(-1)^{q} C_{N_{\mathrm{T}}-1}^{q} \frac{\left(\theta_{1, p}\right)^{q}}{\left(\Omega_{\mathrm{T}_{m-1} \mathrm{~T}_{m}}\right)^{N_{\mathrm{T}}-1-q}} \\
& \times \Gamma\left(N_{\mathrm{T}}-1-q, \theta_{1, p}\right) .
\end{aligned}
$$

Substituting (43) into (42), we obtain $\mathcal{I}$, then combining with (17) and (24), we obtain exact and asymptotic closed-form expressions of the e2e IP of AA$\mathrm{AJ}$ as shown in (44) and (45), respectively.

Remark 5: It is worth noting that when $1-\mu_{\mathrm{E}}^{2} \rho_{\text {th }} \leq 0$, the e2e IP of all the proposed algorithms equals to 0 . Next, as observed from (35), (39) and (45), the e2e IP values at high transmit SNR do not depend on $\Delta$.

\section{Simulation Results}

Section 4 presents Monte-Carlo simulations to verify the derived expressions of the e2e OP and IP. For illustration purpose only, in all the simulations, all the nodes in the $m$-th cluster are placed at $(m / M, 0)$, the position of the eavesdropper is fixed at $(0.5,0.3)$, and the path-loss exponent $(\beta)$ is assigned by 3 , where $m \in$ $[0, M]$. We also assume that the number of members at all the clusters is the same, i.e., $K_{m}=K(\forall m)$. In all the figures, the simulation (Sim) and theoretical (Exact) results match very well, which validates correction of our derivations.

Figs. 2 and 3 respectively present the e2e OP and IP as a function of the transmit SNR $(\Delta)$ in $\mathrm{dB}$ when $M=4, N_{\mathrm{T}}=2, N_{\mathrm{E}}=3, K=4, \alpha=0.1$ and $\mu_{\mathrm{D}}^{2}=\mu_{\mathrm{E}}^{2}=$ 0.05. As shown in Fig. 2, the OP values decrease as $\Delta$ increases because the source and intermediate $\mathrm{CH}$ nodes can transmit the data with higher transmit power. We also see that at high $\Delta$ regimes, the exact OP values nicely converge to the asymptotic ones, and the slope of the OP curves equals to 4 (i.e., $N_{\mathrm{T}}^{2}=4$, see (23)). Moreover, Fig. 2 also presents that the OP performance is better when the target rate $C_{\text {th }}$ decreases. In Fig. 3, the IP values of all the algorithms increase with the increasing of $\Delta$, and they converge to the asymptotic values at high $\Delta$ values (as proved in Remark 5). We can see in Fig. 3 that the IP performance of AA-AJ is best because this method exploits all the antennas and all the available jammers. It is also seen that AA-AJ 


$$
\mathrm{IP}_{\mathrm{e} 2 \mathrm{e}}^{\mathrm{BA}-\mathrm{BJ}}=1-\prod_{m=1}^{M}\left[\begin{array}{c}
1+\sum_{p=1}^{N_{\mathrm{E}}} \sum_{u=0}^{N_{\mathrm{T}} K_{m}-1}(-1)^{p+u} C_{N_{\mathrm{E}}}^{p} C_{N_{\mathrm{T}} K_{m}-1}^{u} N_{\mathrm{T}} K_{m} \exp \left((u+1) \Omega_{\mathrm{T}_{m-1} \mathrm{~T}_{m}} \theta_{1, p}-p \Omega_{\mathrm{T}_{m-1} \mathrm{E}} \omega_{2}\right) \\
\times \Omega_{\mathrm{T}_{m-1} \mathrm{~T}_{m}} \theta_{1, p} E_{1}\left((u+1) \Omega_{\mathrm{T}_{m-1} \mathrm{~T}_{m}} \theta_{1, p}\right)
\end{array}\right]
$$

$$
\mathrm{IP}_{\mathrm{e} 2 \mathrm{e}}^{\mathrm{BA}-\mathrm{BJ}} \stackrel{\Delta \rightarrow+\infty}{\approx} 1-\prod_{m=1}^{M}\left[\begin{array}{c}
1+\sum_{p=1}^{N_{\mathrm{E}}} \sum_{u=0}^{N_{\mathrm{T}} K_{m}-1}(-1)^{p+u} C_{N_{\mathrm{E}}}^{p} C_{N_{\mathrm{T}} K_{m}-1}^{u} N_{\mathrm{T}} K_{m} \exp \left((u+1) \Omega_{\mathrm{T}_{m-1} \mathrm{~T}_{m}} \theta_{1, p}\right) \\
\times \Omega_{\mathrm{T}_{m-1} \mathrm{~T}_{m}} \theta_{1, p} E_{1}\left((u+1) \Omega_{\mathrm{T}_{m-1} \mathrm{~T}_{m}} \theta_{1, p}\right)
\end{array}\right]
$$

$$
\begin{aligned}
& \overline{\mathrm{IP}}_{m}^{\mathrm{RA}-\mathrm{AJ}}=\operatorname{Pr}\left(\psi_{\mathrm{T}_{m-1}^{p}} \mathrm{E}^{r}<\omega_{1} \sum_{u=1}^{K_{m}} \psi_{\mathrm{T}_{m-1}^{c} \mathrm{~J}_{m, u}} \psi_{\mathrm{J}_{m, u}} \mathrm{E}^{r}+\omega_{2}\right) \\
& =1+\sum_{p=1}^{N_{\mathrm{E}}}(-1)^{p} C_{N_{\mathrm{E}}}^{p} \exp \left(-p \Omega_{\mathrm{T}_{m-1} \mathrm{E}} \omega_{2}\right) \prod_{u=1}^{K_{m}} \int_{0}^{+\infty} \int_{0}^{+\infty} \exp \left(-p \Omega_{\mathrm{T}_{m-1} \mathrm{E}} \omega_{1} x y\right) f_{\psi_{\mathrm{J}_{m, u}} \mathrm{E}^{r}}(x) f_{\psi_{\mathrm{T}_{m-1}^{c} \mathrm{~J} m, u}}(y) d x d y .
\end{aligned}
$$

$$
\mathrm{IP}_{\mathrm{e} 2 \mathrm{e}}^{\mathrm{RA}-\mathrm{AJ}}=1-\prod_{m=1}^{M}\left[1+\sum_{p=1}^{N_{\mathrm{E}}}(-1)^{p} C_{N_{\mathrm{E}}}^{p} \exp \left(K_{m} \Omega_{\mathrm{T}_{m-1} \mathrm{~T}_{m}} \theta_{1, p}-p \Omega_{\mathrm{T}_{m-1} \mathrm{E}} \omega_{2}\right)\left[\Omega_{\mathrm{T}_{m-1} \mathrm{~T}_{m}} \theta_{1, p} E_{1}\left(\Omega_{\mathrm{T}_{m-1} \mathrm{~T}_{m}} \theta_{1, p}\right)\right]^{K_{m}}\right]
$$

$$
\mathrm{IP}_{\mathrm{e} 2 \mathrm{e}}^{\mathrm{RA}-\mathrm{AJ}} \stackrel{\Delta \rightarrow+\infty}{\approx} 1-\prod_{m=1}^{M}\left[1+\sum_{p=1}^{N_{\mathrm{E}}}(-1)^{p} C_{N_{\mathrm{E}}}^{p} \exp \left(K_{m} \Omega_{\mathrm{T}_{m-1} \mathrm{~T}_{m}} \theta_{1, p}\right)\left[\Omega_{\mathrm{T}_{m-1} \mathrm{~T}_{m}} \theta_{1, p} E_{1}\left(\Omega_{\mathrm{T}_{m-1} \mathrm{~T}_{m}} \theta_{1, p}\right)\right]^{K_{m}}\right] .
$$

$$
\begin{aligned}
\overline{\mathrm{IP}}_{m}^{\mathrm{RA}-\mathrm{AJ}} & =\operatorname{Pr}\left(\psi_{\mathrm{T}_{m-1}^{p} \mathrm{E}^{r}}<\omega_{1} \sum_{t=1}^{N_{\mathrm{T}}} \sum_{u=1}^{K_{m}} \psi_{\mathrm{T}_{m-1}^{t}} \mathrm{~J}_{m, u} \psi_{\mathrm{J}_{m, u} \mathrm{E}^{r}}+\omega_{2}\right) \\
& =1+\sum_{p=1}^{N_{\mathrm{E}}}(-1)^{p} C_{N_{\mathrm{E}}}^{p} \exp \left(-p \Omega_{\mathrm{T}_{m-1} \mathrm{E}} \omega_{2}\right) \prod_{u=1}^{K_{m}} \int_{0}^{+\infty} \int_{0}^{+\infty} \exp \left(-p \Omega_{\mathrm{T}_{m-1} \mathrm{E}} \omega_{1} x y\right) f_{\psi_{\mathrm{J}_{m, u}} \mathrm{E}^{r}}(x) f_{Y_{\text {sum }}}(y) d x d y \\
& =1+\sum_{p=1}^{N_{\mathrm{E}}}(-1)^{p} C_{N_{\mathrm{E}}}^{p} \exp \left(-p \Omega_{\mathrm{T}_{m-1} \mathrm{E}} \omega_{2}\right) \prod_{u=1}^{K_{m}} \int_{0}^{+\infty} \frac{\theta_{1, p}}{\theta_{1, p}+y} f_{Y_{\text {sum }}}(y) d y .
\end{aligned}
$$

$$
\begin{aligned}
\overline{\mathrm{IP}}_{m}^{\mathrm{AA}-\mathrm{AJ}}=1+ & \sum_{p=1}^{N_{\mathrm{E}}}(-1)^{p} C_{N_{\mathrm{E}}}^{p} \exp \left(K_{m} \Omega_{\mathrm{T}_{m-1} \mathrm{~T}_{m}} \theta_{1, p}-p \Omega_{\mathrm{T}_{m-1} \mathrm{E}} \omega_{2}\right) \\
& \times \prod_{p=1}^{K_{m}}(-1)^{p} \frac{\theta_{1, p} \Omega_{\mathrm{T}_{m-1} \mathrm{~T}_{m}}^{N_{\mathrm{T}}}}{\left(N_{\mathrm{T}}-1\right) !} \underbrace{\int_{\theta_{1, p}}^{+\infty} \frac{1}{z}\left(z-\theta_{1, p}\right)^{N_{\mathrm{T}}-1} \exp \left(-\Omega_{\mathrm{T}_{m-1} \mathrm{~T}_{m}} z\right) d z}_{\mathcal{I}}
\end{aligned}
$$




$$
\mathrm{IP}_{\mathrm{e} 2 \mathrm{e}}^{\mathrm{AA}-\mathrm{AJ}}=1-\prod_{m=1}^{M}\left\{\begin{array}{l}
1+\sum_{p=1}^{N_{\mathrm{E}}}(-1)^{p} C_{N_{\mathrm{E}}}^{p} \exp \left(K_{m} \Omega_{\mathrm{T}_{m-1} \mathrm{~T}_{m}}-p \Omega_{\mathrm{T}_{m-1} \mathrm{E}} \omega_{2}\right) \\
\times \prod_{p=1}^{K_{m}} \frac{1}{\left(N_{\mathrm{T}}-1\right) !}\left[\begin{array}{c}
(-1)^{N_{\mathrm{T}}-1}\left(\theta_{1, p} \Omega_{\mathrm{T}_{m-1} \mathrm{~T}_{m}}\right)^{N_{\mathrm{T}}} E_{1}\left(\Omega_{\mathrm{T}_{m-1} \mathrm{~T}_{m}} \theta_{1, p}\right) \\
+\sum_{q=0}^{N_{\mathrm{T}}-2}(-1)^{q} C_{N_{\mathrm{T}}-1}^{q}\left(\Omega_{\mathrm{T}_{m-1} \mathrm{~T}_{m}} \theta_{1, p}\right)^{q+1} \Gamma\left(N_{\mathrm{T}}-1-q, \Omega_{\mathrm{T}_{m-1} \mathrm{~T}_{m}} \theta_{1, p}\right)
\end{array}\right]
\end{array}\right] .
$$

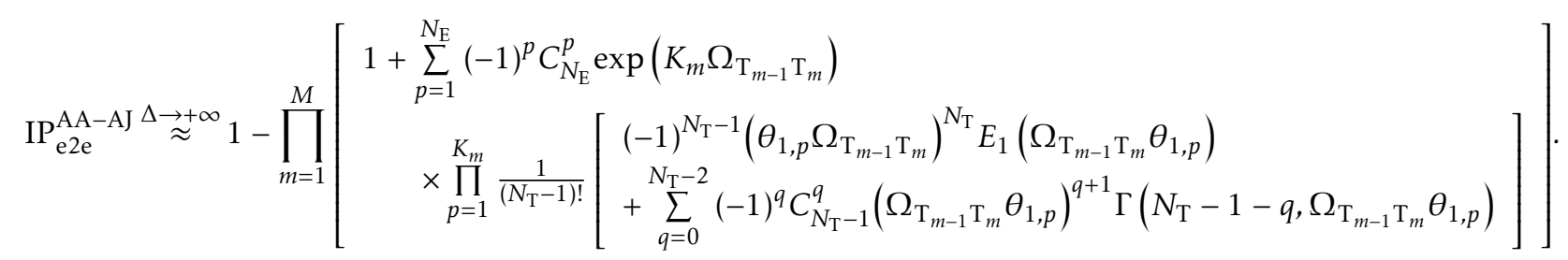

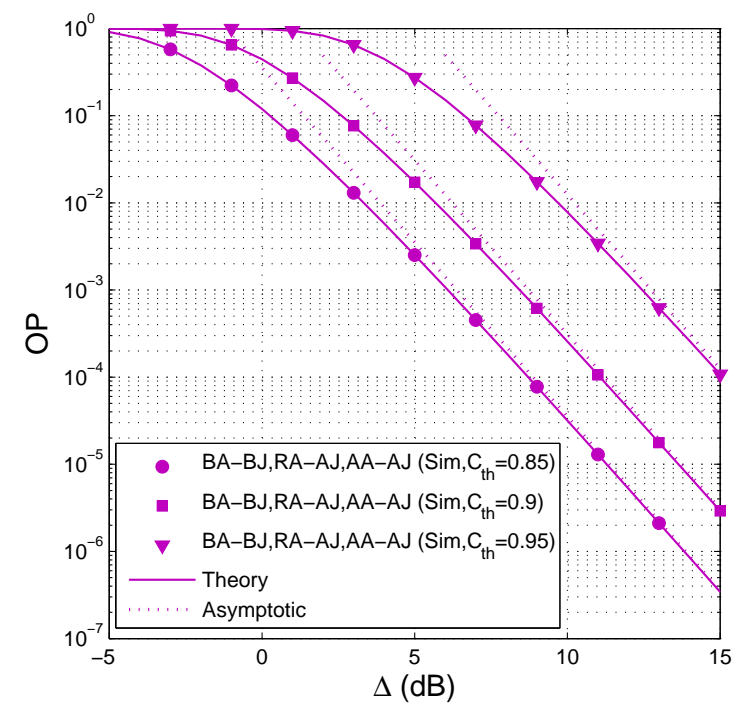

Figure 2. $\mathrm{OP}$ as a function of $\Delta(\mathrm{dB})$ when $M=4, N_{\mathrm{T}}=2$, $K=4, \alpha=0.1$ and $\mu_{\mathrm{D}}^{2}=0.05$.

outperforms BA-BJ, and the IP values increase with the decreasing of $C_{\mathrm{th}}$. From Figs. 2-3, we can see that there exists a trade-off between IP and OP. In particular, to obtain better OP performance with the fixed system parameters, the transmitters in the proposed algorithms must increase transmit power (or increase $\Delta$ ) and/or decrease the target rate $\left(C_{\mathrm{th}}\right)$. However, increasing $\Delta$ and/or decreasing $C_{\text {th }}$ also means increasing IP at the eavesdropper.

Figs. 4 and 5 investigate the impact of the number of hops $(M)$ on the e2e OP and IP performance when $\Delta=5(\mathrm{~dB}), N_{\mathrm{E}}=3, K=3, \alpha=0.1$ and $\mu_{\mathrm{D}}^{2}=\mu_{\mathrm{E}}^{2}=0$. As shown in Fig. 4 , the OP values increase as increasing the number of hops $(M \geq 2)$. It is due to the fact that

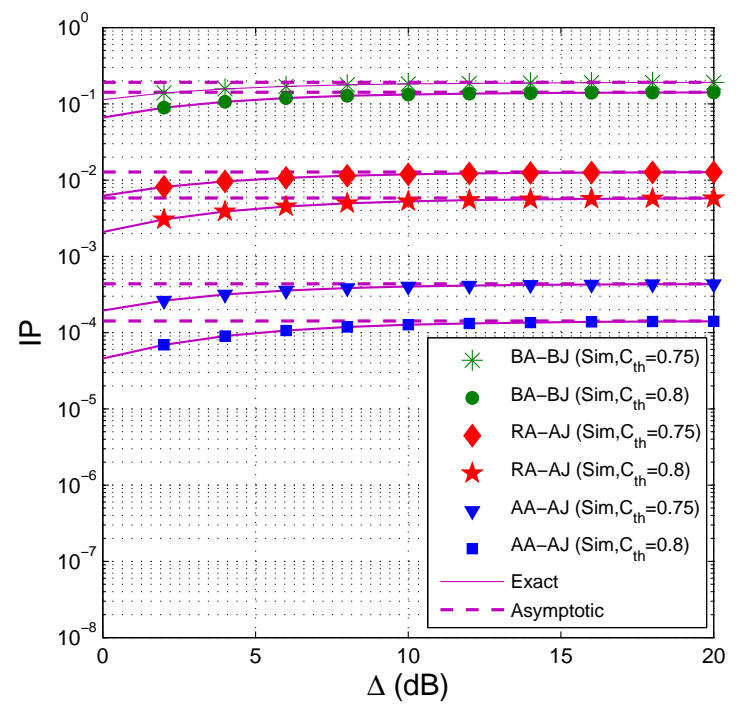

Figure 3. IP as a function of $\Delta(\mathrm{dB})$ when $M=4, N_{\mathrm{T}}=2$, $N_{\mathrm{E}}=3, K=4, \alpha=0.1$ and $\mu_{\mathrm{E}}^{2}=0.05$.

duration of each time slot decreases with the increasing of $M$. In Fig. 4, when $M=1$, the source directly communicates with the destination, without using the relaying technique. Due to the transmission at the far distance, the OP performance with $M=1$ is worse than that with $M=2$. As a result, $M=2$ is shown as the optimal number of hops in Fig. 4. Fig. 4 also illustrates that the OP performance significantly enhances when the number of antennas at the source and $\mathrm{CH}$ nodes changes from 1 to 3 . It is due to the fact that the data transmission at each hop is more reliable with high number of $N_{\mathrm{T}}$. Different with OP, the IP values of all the proposed algorithms in Fig. 5 significantly decrease as increasing the number of hops $(M)$. Again, we can see 


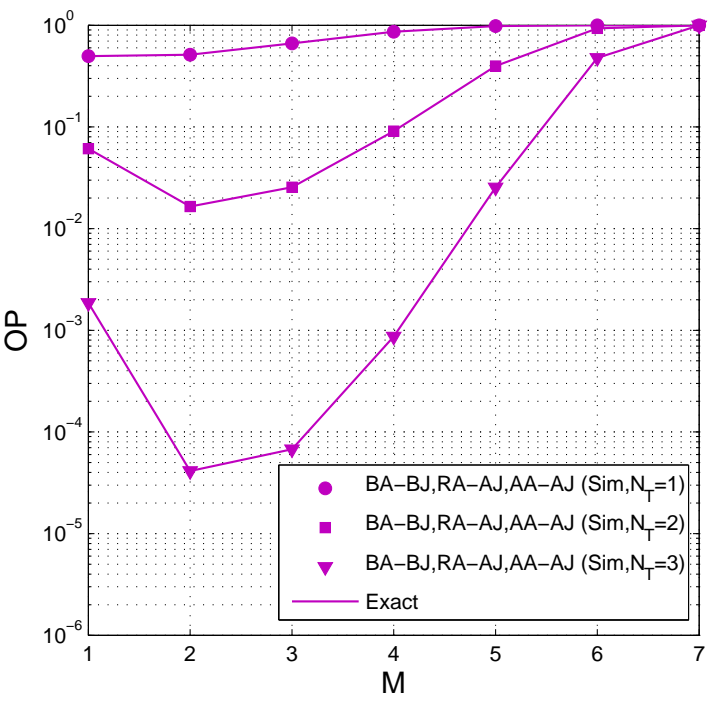

Figure 4. OP as a function of $M$ when $\Delta=5(\mathrm{~dB}), C_{\mathrm{th}}=1.5$, $K=3, \alpha=0.1$ and $\mu_{\mathrm{D}}^{2}=0$.

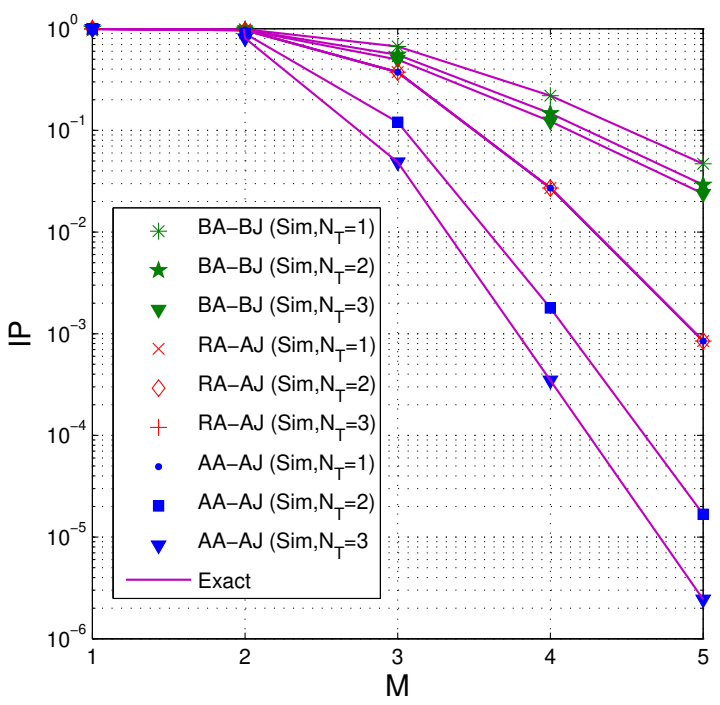

Figure 5. IP as a function of $M$ when $\Delta=5(\mathrm{~dB}), C_{\mathrm{th}}=1$, $N_{\mathrm{E}}=3, K=3, \alpha=0.1$ and $\mu_{\mathrm{E}}^{2}=0$.

that AA-AJ obtains the best IP performance, and RAAJ outperforms BA-BJ. In addition, the IP performance of AA-AJ and BA-BJ is better with the increasing of $N_{\mathrm{T}}$ because more energy can be harvested by the jammer nodes. For RA-AJ, due to the random antenna selection for serving the jammer nodes, the IP values do not depend on $N_{\mathrm{T}}$. It is straightforward that AA-AJ will become RA-AJ when $N_{\mathrm{T}}=1$.

Figs. 6 and 7 study the impact of the HI levels $\mu_{\mathrm{D}}^{2}$ and $\mu_{\mathrm{E}}^{2}$ on the e2e OP and IP performance, respectively, when $\Delta=2.5(\mathrm{~dB}), C_{\mathrm{th}}=0.65, N_{\mathrm{T}}=2, N_{\mathrm{E}}=3, K=4$

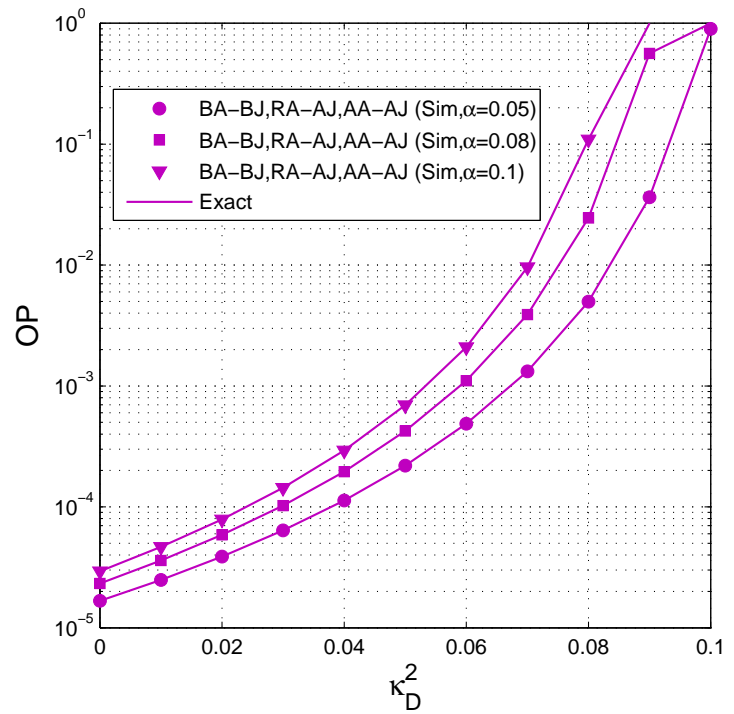

Figure 6. OP as a function of $\mu_{\mathrm{D}}^{2}$ when $\Delta=2.5(\mathrm{~dB}), C_{\mathrm{th}}=$ $0.65, N_{\mathrm{T}}=2, K=4$ and $M=5$.

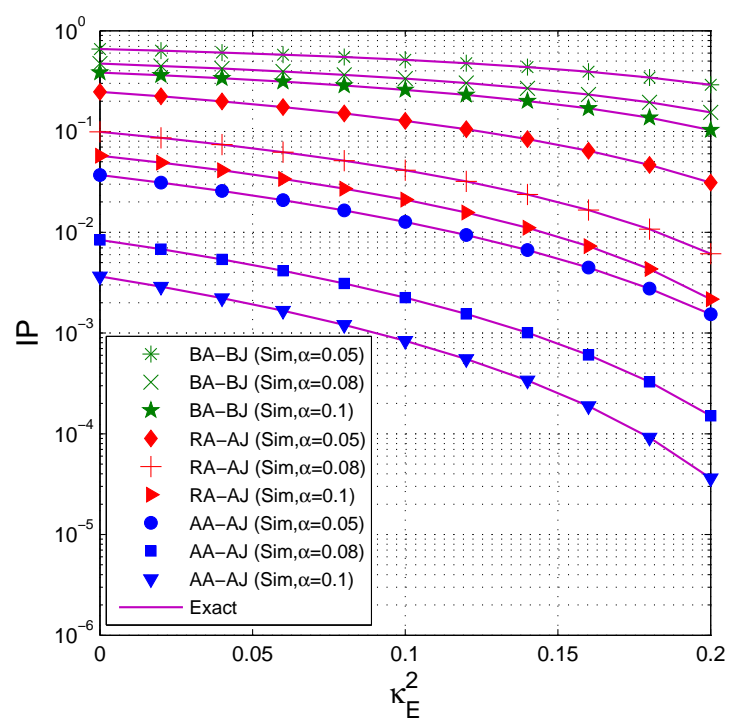

Figure 7. IP as a function of $\mu_{\mathrm{E}}^{2}$ when $\Delta=2.5(\mathrm{~dB}), C_{\mathrm{th}}=$ $0.65, N_{\mathrm{T}}=2, N_{\mathrm{E}}=3, K=4$ and $M=5$.

and $M=5$. As expected, the OP values rapidly increase when $\mu_{\mathrm{D}}^{2}$ increases. Indeed, when $\mu_{\mathrm{D}}^{2}=0.1$, OP almost equals to 1. Fig. 6 also shows that the OP performance is better as $\alpha$ decreases because more time is allocated for the data transfer phase. In Fig. 7, it can be observed that the IP performance is better with higher $\mu_{\mathrm{E}}^{2}$ and $\alpha$ values. From Figs. 6 and 7, it is noted that the fraction of time $\alpha$ should be carefully designed to obtain the desired OP or IP performance.

Fig. 8 shows the e2e IP as a function of $K$ when $\Delta=5$ (dB), $C_{\text {th }}=0.5, N_{\mathrm{T}}=2, \alpha=0.1, K=4, M=5$ and $\mu_{\mathrm{E}}^{2}=$ 


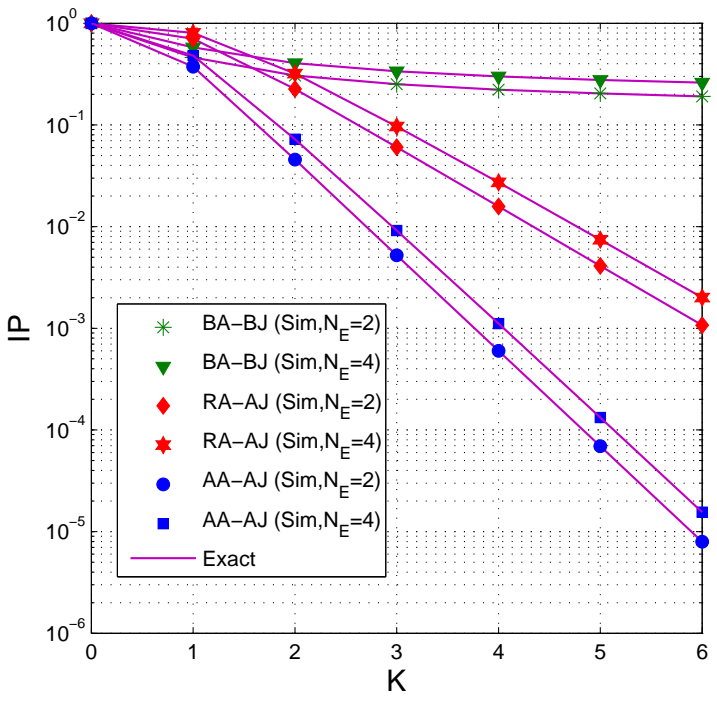

Figure 8. IP as a function of $K$ when $\Delta=5(\mathrm{~dB}), C_{\mathrm{th}}=0.5$, $N_{\mathrm{T}}=2, \alpha=0.1, K=4, M=5$ and $\mu_{\mathrm{E}}^{2}=0$.

0 . We note that the value of $K$ does not impact on the e2e OP performance, and hence the OP performance is not presented in this figure. We can see that IP of BA-BJ, RA-AJ and AA-AJ significantly decreases as increasing $K$. When $C$ J is not used $(K=0)$, we can see that IP of all the algorithms almost equals to 1 . With $K=1$, the IP performance of BA-BJ is better than that of RA-AJ due to the best antenna selection used in BA-BJ. However, when $K \geq 2$, RA-AJ obtains better IP performance, as compared with BA-BJ. Again, AA-AJ provides the best IP performance, and the performance gaps between AA$\mathrm{AJ}$ and two remaining algorithms rapidly increase when $K$ increases. Finally, as expected, the IP performance is worse as the E node is equipped with higher antennas.

Figs. 9-10 present the IP-OP trade-off performance. In these figures, the values of the e2e OP are first determined, and are denoted by $\mathrm{OP}_{\text {target }}$, i.e., $10^{-4} \leq$ $\mathrm{OP}_{\text {target }} \leq 10^{-1}$ as in Figs. 9-10. Next, with the set-up system parameters (see below Figs. 9-10), we solve the equations $\mathrm{OP}_{\mathrm{e} 2 \mathrm{e}}=\mathrm{OP}_{\text {target }}$ to find the corresponding values of $\Delta$. Then, we use the derived formulas (33), (38) and (44) to obtain the values of IP, and present IP as a function of OP. We first see from Figs. 9-10 that as OP decreases, the corresponding IP increases, which shows the trade-off between security and reliability. As mentioned above, to obtain lower OP, the transmitters have to use higher transmit power which also increases IP. Next, because all the algorithms have the same OP value, IP of AA-AJ is lowest, and IP of RA-AJ is between those of BA-BJ and AA-AJ, as illustrated in Figs. 910. Fig. 9 presents more clearly that with the same OP value, the IP value significantly as increasing the number of the jammer nodes $(K)$. In Fig. 10, we can

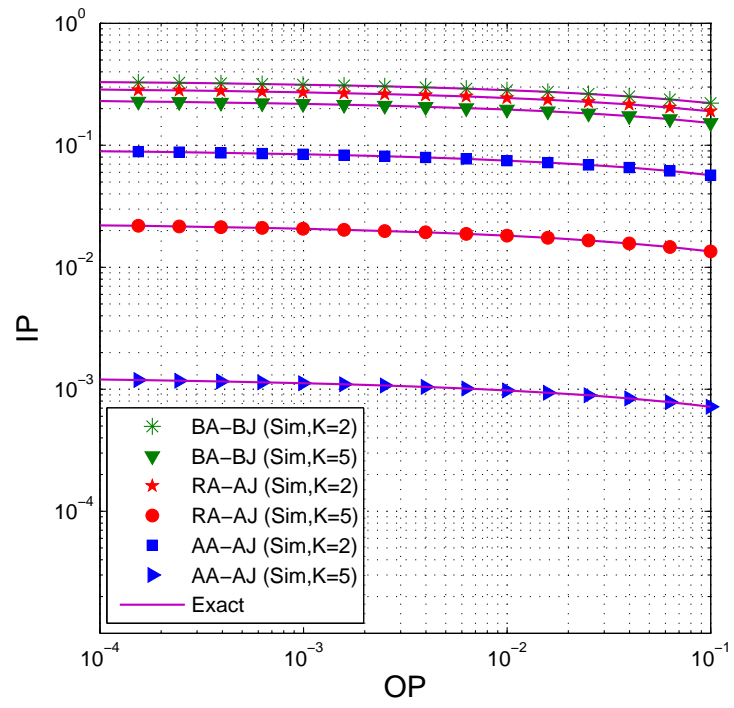

Figure 9. IP as a function of $\mathrm{OP}$ when $C_{\mathrm{th}}=1.25, N_{\mathrm{T}}=2$, $N_{\mathrm{E}}=2, \alpha=0.1, M=3$ and $\mu_{\mathrm{E}}^{2}=\mu_{\mathrm{D}}^{2}=0.01$.

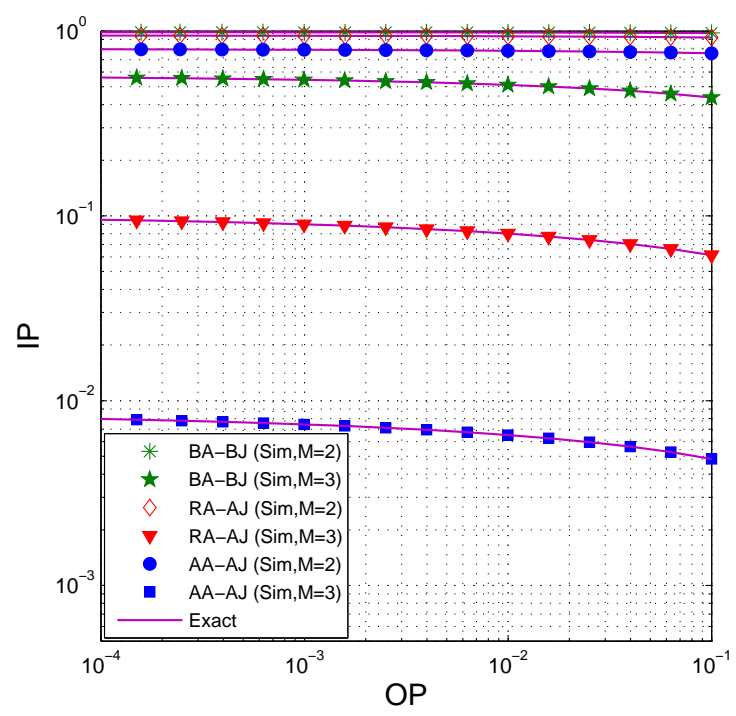

Figure 10. IP as a function of $\mathrm{OP}$ when $C_{\mathrm{th}}=0.9, N_{\mathrm{T}}=2$, $N_{\mathrm{E}}=4, \alpha=0.1, K=7$ and $\mu_{\mathrm{E}}^{2}=\mu_{\mathrm{D}}^{2}=0.01$.

see that the IP values significantly decreases when $M$ changes from 2 to 3 .

\section{Conclusions}

This paper proposed three efficient CJ algorithms to enhance the secrecy performance of the harvest-to-jam based multi-hop cluster MIMO networks, in terms of the e2e IP. In addition, we derived exact expressions of the e2e OP and IP for the proposed BA-BJ, RA-AJ and AA-AJ algorithms, which were verified the correction by computer simulations. The obtained results showed 
that all the algorithms have the same OP performance, and the IP performance of AA-AJ is much better than that of BA-BJ and RA-AJ. However, the implementation of AA-AJ is most complex. The results also presented the key role of the CJ technique in the PLS networks. Moreover, increasing the number of the jammer nodes significantly enhances the IP performance. Besides the security-complexity trade-off, the security-reliability trade-off was investigated. This paper showed that the SRT performance can be enhanced by increasing the number of hops and the number of jammer nodes.

\section{References}

[1] G. Han, H. Xu, T. Q. Duong, J. Jiang and T. Hara, "Localization Algorithms of Wireless Sensor Networks: A Survey," Telecommunication Systems, vol. 52, no. 4, pp. 2419-2436, Apr. 2013.

[2] G. Han, J. Jiang, C. Zhang, T. Q. Duong, M. Guizani and G. K. Karagiannidis, "A Survey on Mobile Anchor Node Assisted Localization in Wireless Sensor Networks," IEEE Communications Surveys \& Tutorials, vol. 18, no. 3, pp. 2220-2243, Third Quarter 2016.

[3] P. N. Son, D. Har, N. I. Cho and H. Y. Kong, "Optimal Power Allocation Of Relay Sensor Node Capable Of Energy Harvesting In Cooperative Cognitive Radio Network," Sensors, vol. 17, no. 3, pp. 1-18, Mar. 2017.

[4] T. D. Hieu, T. T. Duy and B.-S. Kim, "Performance Enhancement for Multi-hop Harvest-to-Transmit WSNs With Path-Selection Methods in Presence of Eavesdroppers and Hardware Noises," IEEE Sensors Journal, vol. 18, no. 12, pp. 5173-5186, Jun. 2018.

[5] P. Zhang, A. Y. Gao and O. Theel, "Bandit Learning with Concurrent Transmissions for Energy-Efficient Flooding in Sensor Networks," EAI Endorsed Transactions on Industrial Networks and Intelligent Systems, vol. 4, no. 13, pp. 1-14, Mar. 2018.

[6] R. E. Mohamed, A. I. Saleh, M. Abdelrazzak and A. S. Samra, "Survey on Wireless Sensor Network Applications and Energy Efficient Routing Protocols," Wireless Personal Communications, vol. 101, no. 2, pp. 1019-1055, May 2018.

[7] H. Yetgin, K. T. K. Cheung, M. El-Hajjar and L. Hanzo, "A Survey of Network Lifetime Maximization Techniques in Wireless Sensor Networks," IEEE Communications Surveys E Tutorials, vol. 19, no. 2, pp. 828-854, Jan. 2017.

[8] N. A. Pantazis, S. A. Nikolidakis and D. D. Vergados, "Energy-Efficient Routing Protocols in Wireless Sensor Networks: A Survey," IEEE Communications Surveys \& Tutorials, vol. 15, no. 2, pp. 551-591, Second Quarter 2013.

[9] H. El Alami and A. Najid, "ECH: An Enhanced Clustering Hierarchy Approach to Maximize Lifetime of Wireless Sensor Networks," IEEE Access, vol. 7, pp. 107142107153, Aug. 2019.

[10] S. K. Singh, P. Kumar and J. P. Singh, "A Survey on Successors of LEACH Protocol," IEEE Access, vol. 5, pp. 4298-4328, Feb. 2017.

[11] G. Farhadi and N. C. Beaulieu, "On the Performance Of Amplify-and-Forward Cooperative Systems With
Fixed Gain Relays," IEEE Transactions on Wireless Communications, vol. 7, no. 5, pp. 1851-1856, May 2008.

[12] H. V. Khuong and P. C. Sofotasios, "Exact Bit-ErrorRate Analysis Of Underlay Decode-and-Forward Multihop Cognitive Networks With Estimation Errors," IET Communications, vol. 7, no. 18, pp. 2122-2132, Dec. 2013.

[13] H. V. Khuong, "Impact Of Imperfect Channel Information On The Performance Of Underlay Cognitive DF Multi-Hop Systems," Wireless Personal Communications, vol. 74, no. 2, pp. 487-498, Jan. 2014.

[14] F. S. Al-Qahtani, R. M. Radaydeh, S. Hessien, T. Q. Duong and H. Alnuweiri, "Underlay Cognitive Multihop MIMO Networks With and Without Receive Interference Cancellation," IEEE Transactions on Communications, vol. 65, no. 4, pp. 1477-1493, Apr. 2017.

[15] Q. N. Tran, N. S. Vo, Q. A. Nguyen, M. P. Bui, T. M. Phan, V. V. Lam and A. Masaracchia, "D2D Multi-hop Multi-path Communications in B5G Networks: A Survey on Models, Techniques, and Applications," EAI Endorsed Transactions on Industrial Networks and Intelligent Systems, vol. 7, no. 25, pp. 1-12, Jan. 2021.

[16] J. Yao, X. Zhou, Y. Liu and S. Feng, "Secure Transmission in Linear Multihop Relaying Networks," IEEE Transactions on Wireless Communications, vol. 17, no. 2, pp. 822-834, Feb. 2018.

[17] K. Zheng, X. Liu, L. Fu, X. Wang and Y. Zhu, "Energy Efficiency in Multihop Wireless Networks with Unreliable Links," IEEE Transactions on Network Science and Engineering, vol. 7, no. 1, pp. 576-588, Jan.-Mar. 2020.

[18] R. Katla and A.V. Babu, "Multihop Full Duplex Relaying With Coherent Signaling: Outage Probability Analysis and Power Optimization," Ad-hoc Networks, vol.97, pp.113, Feb. 2020.

[19] P. T. Tin, D. T. Hung, N. N. Tan, T. T. Duy and M. Voznak, "Secrecy Performance Enhancement for Underlay Cognitive Radio Networks Employing Cooperative Multihop Transmission With and Without Presence of Hardware Impairments," Entropy MDPI, vol. 21, no. 2, pp. 1-16, Feb. 2019.

[20] P. M. Nam, T. T. Duy, P. V. Ca, P. N. Son and N. H. An, "Outage Performance of Power Beacon-Aided Multi-Hop Cooperative Cognitive Radio Protocol Under Constraint of Interference and Hardware Noises," Electronics MDPI, vol. 9, no. 6, pp. 1-19, Jun. 2020.

[21] J. N. Laneman, D. N. Tse, and G. W. Wornell, "Cooperative Diversity in Wireless Networks: Efficient Protocols and Outage Behavior," IEEE Transactions on Information Theory, vol. 5, no. 12, pp. 3062-3080, Dec. 2004.

[22] M. R. Bhatnagar, "Performance Analysis Of a Path Selection Scheme in Multi-hop Decode-and-Forward Protocol," IEEE Communications Letters, vol. 16, no. 12, pp. 1980-1983, Dec. 2012.

[23] M. R. Bhatnagar, R. K. Mallik and O. Tirkkonen, "Performance Evaluation of Best-Path Selection in a Multihop Decode-and-Forward Cooperative System," IEEE Transactions on Vehicular Technology, vol. 65, no. 4, pp. 2722-2728, Apr. 2016.

[24] T. T. Duy, P. T. D. Ngoc and T. T. Phuong, "Performance Enhancement for Multihop Cognitive DF and AF Relaying Protocols under Joint Impact of Interference 
and Hardware Noises: NOMA for Primary Network and Best-Path Selection for Secondary Network," Wireless Communications and Mobile Computing, vol. 2021, ID 8861725, pp. 1-15, Apr. 2021.

[25] H. K. Boddapati, M. R. Bhatnagar and S. Prakriya, "Performance Analysis of Cluster-Based Multi-Hop Underlay CRNs Using Max-Link-Selection Protocol," IEEE Transactions on Cognitive Communications and Networking, vol. 4, no. 1, pp. 15-29, Mar. 2018.

[26] H. K. Boddapati, M. R. Bhatnagar and S. Prakriya, "Performance of Incremental Relaying Protocols for Cooperative Multihop CRNs," IEEE Transactions on Vehicular Technology, vol. 67, no. 7, pp. 6006-6022, Jul. 2018.

[27] P. N. Son and H. Y. Kong, "Cooperative Communication With Energy Harvesting Relays Under Physical Layer Security," IET Communications, vol. 9, no. 17, pp. 21312139, Nov. 2015.

[28] G. Pan, H. Lei, Y. Yuan and Z. Ding, "Performance Analysis and Optimization for SWIPT Wireless Sensor Networks," IEEE Transactions on Communications, vol. 65, no. 5, pp. 2291-2302, May 2017.

[29] A. A. Nasir, H. D. Tuan, T. Q. Duong and M. Debbah, "NOMA Throughput and Energy Efficiency in Energy Harvesting Enabled Networks," IEEE Transactions on Communications, vol. 67, no. 9, pp. 6499-6511, Sept. 2019.

[30] N. N. Tan, T. H. Q. Minh, T. T. Phuong, M. Voznak, T. T. Duy, N. T. Long and P. T. Tin, "Performance Enhancement for Energy Harvesting Based Two-Way Relay Protocols in Wireless Ad-hoc Networks with Partial and Full Relay Selection Methods," Ad-Hoc Networks, vol. 54, pp. 178187, Mar. 2019.

[31] C. Yin, H. T. Nguyen, C. Kundu, Z. Kaleem, E. GarciaPalacios and T. Q. Duong, "Secure Energy Harvesting Relay Networks With Unreliable Backhaul Connections," IEEE Access, vol. 6, pp. 12074-12084, Jan. 2018.

[32] V. N. Vo, T. G. Nguyen, C. So-in, Z. A. Baig, and S. Sanguanpong, "Secrecy Outage Performance Analysis for Energy Harvesting Sensor Networks With a Jammer Using Relay Selection Strategy," IEEE Access, vol.6, pp. 2340623419, May. 2018

[33] K. N. Le and T. A. Tsiftsis, "Wireless Security Employing Opportunistic Relays and an Adaptive Encoder Under Outdated CSI and Dual-Correlated Nakagami- $m$ Fading," IEEE Transactions on Communications, vol. 67, no. 3, pp. 2405-2419, Mar. 2019.

[34] H. V. Khuong and D. D. Thiem, "Relay Selection for Security Improvement in Cognitive Radio Networks with Energy Harvesting," Wireless Communications and Mobile Computing, vol. 2021, pp. 1-16, Jun. 2021.

[35] L. J. Rodriguez, N. H. Tran, T. Q. Duong, T. Le-Ngoc, M. Elkashlan and S. Shetty, "Physical Layer Security In Wireless Cooperative Relay Networks: State Of the Art and Beyond," IEEE Communications Magazine, vol. 53, no. 12, pp. 32-39, Dec. 2015.

[36] X. Jiang, C. Zhong, X. Chen, T. Q. Duong, T. A. Tsiftsis and Z. Zhang, "Secrecy Performance of Wirelessly Powered Wiretap Channels," IEEE Transactions on Communications, vol. 64, no. 9, pp. 3858-3871, Sept. 2016.
[37] J. Zhang, T. Q. Duong, R. Woods and A. Marshall, "Securing Wireless Communications of the Internet of Things from the Physical Layer, An Overview," Entropy MDPI, vol. 19, no. 8, pp. 1-16, Aug. 2017.

[38] T. T. Duy, T. Q. Duong, T. L. Thanh and V. N. Q. Bao, "Secrecy Performance Analysis with Relay Selection Methods under Impact of Co-channel Interference," IET Communications, vol. 9, no. 11, pp. 1427-1435, Jul. 2015.

[39] L. Fan, X. Lei, N. Yang, T. Q. Duong and G. K. Karagiannidis, "Secure Multiple Amplify-and-Forward Relaying With Cochannel Interference," IEEE Journal of Selected Topics in Signal Processing, vol. 10, no. 8, pp. 14941505, Dec. 2016.

[40] N. Q. Sang, N. T. Huy, D. D. Van and W.-J. Hwang, "Exact Outage Analysis of Cognitive Energy Harvesting Relaying Networks under Physical Layer Security," EAI Endorsed Transactions on Industrial Networks and Intelligent Systems, vol. 6, no. 18, pp. 1-14, Mar. 2019.

[41] T. T. Truc, "Network-Coding-based Jamming With Triple Transmission Time Slots: A Method To Secure Transmission In An Extreme Case of Source-Wiretapping and Unshared Jamming Signal," EAI Endorsed Transactions on Industrial Networks and Intelligent Systems, vol. 8, no. 27, pp. 1-16, Jun. 2021.

[42] Y. Zou, B. Champagne, W. Zhu and L. Hanzo, "RelaySelection Improves the Security-Reliability Trade-Off in Cognitive Radio Systems," IEEE Transactions on Communications, vol. 63, no. 1, pp. 215-228, Jan. 2015.

[43] Y. Zou, J. Zhu, X. Li and L. Hanzo, "Relay Selection for Wireless Communications Against Eavesdropping: a Security-Reliability Trade-off Perspective," IEEE Network, vol. 30, no. 5, pp. 74-79, Sept.-Oct. 2016.

[44] Y. Liu, L. Wang, T. T. Duy, M. Elkashlan and T. Q. Duong, "Relay Selection for Security Enhancement in Cognitive Relay Networks," IEEE Wireless Communications Letters, vol. 4, no. 1, pp. 46-49, Feb. 2015.

[45] T. M. Hoang, T. Q. Duong, N.-S. Vo and C. Kundu, "Physical Layer Security in Cooperative Energy Harvesting Networks With a Friendly Jammer," IEEE Wireless Communications Letters, vol. 6, no. 2, pp. 174-177, Apr. 2017.

[46] V. L. Nguyen, H. D. Binh, T. D. Dung and Y. Lee, "Enhancing Physical Layer Security for Cooperative NonOrthogonal Multiple Access Networks with Artificial Noise," EAI Endorsed Transactions on Industrial Networks and Intelligent Systems, vol. 6, no. 20, pp. 1-11, Aug. 2019.

[47] K. Cao, B. Wang, H. Ding, L. Lv, J. Tian and F. Gong, "On the Security Enhancement of Uplink NOMA Systems With Jammer Selection," IEEE Transactions on Communications, vol. 68, no. 9, pp. 5747-5763, Sept. 2020.

[48] X. Ding, T. Song, Y. Zou and X. Chen, "SecurityReliability Tradeoff for Friendly Jammer Assisted UserPair Selection in the Face of Multiple Eavesdroppers," IEEE Access, vol. 4, pp. 8386-8393, Sept. 2016.

[49] X. Ding, T. Song, Y. Zou, X. Chen and L. Hanzo, "Security-Reliability Tradeoff Analysis of Artificial Noise Aided Two-Way Opportunistic Relay Selection," IEEE Transactions on Vehicular Technology, vol. 66, no. 5, pp. 3930-3941, May 2017. 
[50] N. T. Anh, T. T. Duy, H. D. Hung, H. D. Hai and N. C. Minh, "Reliability-Security Analysis for Harvest-toJam based Multi-hop LEACH Networks under Impact of Hardware Noises," in proc. of the 2019 International Conference on Advanced Technologies for Communications (ATC 2019), pp. 174-178, Oct. 2019.

[51] D. T. Hung, T. T. Duy and D. Q. Trinh, "SecurityReliability Analysis of Multi-hop LEACH Protocol with Fountain Codes and Cooperative Jamming," EAI Transactions on Industrial Networks and Intelligent Systems, vol. 6, no. 18, pp. 1-7, Mar. 2019.

[52] P. T. Tin, P. M. Nam, T. T. Duy, T. T. Phuong and M. Voznak, "Secrecy Performance of TAS/SC-based Multihop Harvest-to-Transmit Cognitive WSNs under Joint
Constraint of Interference and Hardware Imperfection," Sensors MDPI, vol. 19, no. 5, pp. 1-20, March 2019.

[53] H. D. Hung, T. T. Duy and M. Voznak, "Secrecy Outage Performance of Multi-hop LEACH Networks using Power Beacon Aided Cooperative Jamming with Jammer Selection Methods," AEU - International Journal of Electronics and Communications, vol. 124, ID 153357, pp. 1-25, Sept. 2020.

[54] I. S. Gradshteyn and I. M. Ryzhik, "Table of Integrals, Series, and Products," Academic press, 2014.

[55] S. V. Amari and R. B. Misra, "Closed-Form Expressions For Distribution Of Sum Of Exponential Random Variables," IEEE Transactions on Reliability, vol. 46, no. 4, pp. 519-522, Dec. 1997. 